\title{
SINTAXIS MEDIEVAL IBERORROMÁNICA: LA ESTRUCTURA DE CONSTRUCCIONES CON VERBO REGENTE E INFINITIVO EN TEXTOS ESPAÑOLES Y PORTUGUESES MEDIEVALES*
}

\section{TIBOR BERTA}

\author{
Hispanisztika Tanszék \\ Neolatin Kultúrák Intézete \\ Bölcsészettudományi Kar \\ Szegedi Tudományegyetem \\ Petőfi sgt. 30-34. \\ H-6722 Szeged \\ tberta@hist.u-szeged.hu
}

Spanish and Portuguese, the two most representative Iberoromance languages, coincide in having preserved the phenomenon of clitic promotion since ancient times. According to certain theories, the realization or non-realization of the promotion depend on the structural characteristics of the constructions made up of a regent verb and a dependent infinitive. The detailed analysis of the phenomenon mentioned above in a medieval and a modern corpus presented in this article demonstrates that, in spite of the apparent similarities, there are considerable differences between the structure of the constructions composed of a regent verb and an infinitive in the two periods.

\section{INTRODUCCIÓN}

El español y el portugués, las dos lenguas más representativas de la llamada Iberorromania coinciden en el hecho de que tanto en sus variantes medievales como en sus variantes actuales existen construcciones constituidas por un verbo regente y un infinitivo dependiente en las que los pronombres clíticos que representan complementos - objeto directo o indirecto - del segundo verbo no sólo pueden apoyarse en éste sino también en aquél; cf. (1) y (2). En este último caso se trata del fenómeno de la llamada promoción de clíticos. ${ }^{1}$

* Agradezco los comentarios y observaciones del Prof. Dr. Giampaolo Salvi (Universidad Loránd Eötvös de Budapest). Naturalmente, todos los errores que aparezcan son de mi absoluta responsabilidad.

${ }^{1}$ La alternancia de las dos posiciones y el fenómeno de la promoción de clíticos han sido abundantemente estudiados en español por una larga serie de autores, entre ellos Bolinger 
(1) a. esp. act. Juan puede hacerlo.

b. ptg. act. O João pode fazê-lo.

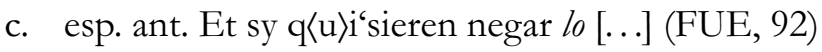

d. ptg. ant. [...] e nom ousei a prová-la. (GRA, 33)

(2) a. esp. act. Juan lo puede hacer.

b. ptg. act. O João não $o$ pode fazer.

c. esp. ant. yo quiero gelo dar. (VEI, 45)

d. ptg. ant. quero-lhe tolher a coroa (GRA, 64)

e. esp. ant. assi la podedes cobrar por el (ULT, 7)

f. ptg. ant. E, quando o assy vyo, non $o$ quis matar. (CGE, 31)

Las teorías modernas atribuyen la aparición de las dos secuencias sintácticas mencionadas a ciertas diferencias estructurales que existen entre los dos tipos de construcción. La teoría de la restructuración propuesta por Rizzi (1982) para el caso del italiano moderno admite que el clítico debe encontrarse en la misma frase en la que se sitúa el infinitivo al que pertenece y supone que cuando el clítico no se aleja del infinitivo, los dos verbos funcionan como núcleos de dos frases distintas - como en (3a) -, pero cuando se produce la promoción, los dos verbos llegan a formar una unidad verbal compleja como en $(3 \mathrm{~b})$ - a consecuencia de una restructuración. ${ }^{2}$

(3) a. esp. act. [Juan [V debe] [F decírtelo ] ]

b. esp. act. [F Juan te lo [V debe decir ] ]

Aplicando los términos utilizados por Benucci (1990) se puede decir que en (3a) la construcción es "difrástica", mientras que en (3b) es "monofrástica".

El objetivo de este trabajo es demostrar que la restructuración puede ser considerada condición de la promoción de clíticos en el caso de las variantes actuales del español y del portugués pero no en el caso de sus variantes medievales y que por ello se deben atribuir estructuras distintas a las construcciones

(1949); Colburn (1928); Davies (1995; 1997b); Fernández (2000); Luján (1993); Pizzini (1982); Rivero (1991; 1993); Spaulding (1927) y Wanner (1982). Las condiciones de la misma alternancia en el portugués han sido analizadas por Pizzini (1981) y Quicoli (1976) mientras que Davies (1997a) se ha dedicado a la historia del mismo fenómeno en portugués. Nosotros también hemos dedicado algunas páginas a la misma cuestión examinando la promoción de clíticos en español sincrónicamente en Berta (2000a) y diacrónicamente en Berta (2000b), mientras que en Berta (2001) se ha examinado la situación medieval del español y del portugués.

${ }^{2}$ Nótese que esta unidad verbal restructurada no corresponde a la unidad entendida como perífrasis verbal porque sólo se refiere al caso de (3b), mientras que el concepto tradicional de la perífrasis no hace distinción entre (3a) y (3b). 
de infinitivo modernas y medievales, respectivamente. Nuestra hipótesis se basa en la comparación de ejemplos modernos construidos por nosotros y comprobados por hablantes nativos ${ }^{3}$ con ejemplos antiguos registrados en textos procedentes de los siglos XIII-XV. El análisis se limita a aquellas construcciones en las que el pronombre clítico representa un complemento regido por el infinitivo, es decir, se excluirán las construcciones causativas y con verbos de percepción, en las cuales el clítico se asocia con el sujeto del infinitivo dependiente - del tipo los hice salir y los vi llegar, respectivamente.

\section{LA TEORÍA DE LA RESTRUCTURACIÓN EN ESPAÑOL $Y$ PORTUGUÉS}

\subsection{El caso del infinitivo antepuesto}

Como se ha mencionado, la teoría de la restructuración supone que la promoción de clíticos es posible porque el verbo regente y el infinitivo forman una unidad sintáctica restructurada. Asimismo, cuando las circunstancias sintácticas demuestran que los dos verbos no constituyen una unidad, la promoción no puede producirse. De acuerdo con ello, no es posible anteponer al verbo regente el constituyente que contiene el infinitivo cuando se produce la restructuración y, gracias a ésta, la promoción de clíticos. Esta idea se puede ilustrar en las fórmulas que aparecen en (4a) y (4b). En (4a) el infinitivo (V) y su complemento $(\mathrm{X})$ forman un constituyente $(\mathrm{S})$, que puede anteponerse al verbo regente (Aux). En (4b), sin embargo, el conjunto $Z$, formado por $V$ y X, no puede desplazarse debido a que $\mathrm{V}$ forma un constituyente con Aux y no con X. Los ejemplos del español actual que se presentan en (4c-e), que corresponden a los ejemplos italianos aducidos por Rizzi (1982: 6-7), ilustran esta situación.

(4) a. Aux $[\mathrm{s} V \mathrm{X}] \rightarrow[\mathrm{s} V \mathrm{X}]$ Aux

b. $[\operatorname{Aux} \underset{\mathrm{Z}}{\mathrm{V}}] \mathrm{X} \rightarrow * \underset{\mathrm{Z}}{\mathrm{V}} \mathrm{X}$ Aux

c. Estos problemas, hablarte de los cuales quiero más tarde...

d. *Estos problemas, hablar de losó cuales te quiero más tarde...

e. Estos problemas, de los cuales te quiero hablar más tarde...

De acuerdo con lo expuesto, (4c) es gramatical debido a que tiene una estructura correspondiente a (4a), mientras que (4d) es agramatical porque la

\footnotetext{
${ }^{3}$ Una parte de las frases modernas son reproducciones de los ejemplos italianos de Rizzi. En estos casos se indicará su procedencia.
} 
anteposición de la unidad que contiene el infinitivo - hablar de los cuales - sólo es posible si éste y el verbo regente no forman una unidad, sin embargo en este caso el clítico no debería haberse colocado delante de la forma verbal quiero. En los textos medievales la anteposición del infinitivo no impide la promoción de clíticos, como demuestran los ejemplos de (5).

(5) a. esp. ant. [...] \& el pescado que $\mathrm{p}\langle\mathrm{r}\rangle \mathrm{i}^{\text {isiere }}$ pecharlo aa su due $\langle\mathrm{n}\rangle \mathrm{no}$. (FUE, 80)

b. ptg. ant. Leixar-nos queredes a tal festa [...]? (GRA, 20)

c. esp. ant. ca de partir vos quiero yo lo que [...] (VEI, 15)

d. esp. ant. Que dixieran del Theodosio si alcançar le mereçieran? (ARA, 20)

e. esp. ant. Et si saluar non se $\mathrm{q}\langle\mathrm{u}\rangle \mathrm{i}^{\prime}$ siere deue gela pechar. (FUE, 91)

f. ptg. ant. Conffessar no $\langle\mathrm{m}\rangle$ sse deue ne $\langle\mathrm{m}\rangle$ huu$\langle\mathrm{m}\rangle$ se [...] (SET, 129)

g. esp. ant. [...] el propheta Hieremias conparar ni significar no la sabe [...] (ARA, 50)

h. esp. ant. [...] y mayor procurar no le podemos que sacar delas manos del ingrato y rebelde oluido [...] (ARA, 42)

Desde el punto de vista de la hipótesis inicial que defendemos se deben tener en cuenta dos problemas relacionados con tales ejemplos. Por una parte, nótese que la ortografía en el ejemplo español de (5a), así como en el ejemplo portugués aducido en (5b), presentado con las normas ortográficas de la edición modernizada utilizada durante el análisis, sugieren que en estos casos el infinitivo y el clítico han sido desplazados juntos. En (5c-d), por otra parte, la ortografía medieval no aclara si se ha producido la promoción de clíticos o no. Teóricamente se puede suponer que en todos estos casos el clítico y el infinitivo han sido movidos juntos, lo cual significa que no se ha producido la promoción del pronombre átono. Los ejemplos presentados en ( $5 \mathrm{e}-\mathrm{h})$, sin embargo, demuestran la posibilidad de que el infinitivo sea movido independientemente del clítico. En ellos el clítico y el infinitivo están separados por el adverbio negativo - non y no, respectivamente -, lo cual es prueba de que el infinitivo y el clítico han sido movidos independientemente. El otro problema mencionado es que el mero desplazamiento por separado del infinitivo y del clítico demuestra únicamente que la anteposición de aquél no bloquea la promoción pero en realidad no contradice la posibilidad de la restructuración. Nótese que Rizzi demuestra que, de acuerdo con (4b), el infinitivo (V) y su complemento $(\mathrm{X})$ no pueden desplazarse juntos cuando aquél pertenece a una unidad verbal restructurada [Aux V], pero sostiene que esta unidad no se 
comporta como una categoría léxica. ${ }^{4}$ Si se acepta esta afirmación, se puede suponer también que los dos componentes del complejo verbal pueden extraerse de él independientemente, es decir, no está prohibido que sólo V se anteponga a Aux abandonando la unidad restructurada, más o menos como aparece en la siguiente fórmula: [Aux V] $\rightarrow \mathrm{V}$ [Aux _ ]. Admitiendo esta posibilidad, (5e-g) no contradicen la teoría de la restructuración. En (5h), sin embargo, en que también se produce la promoción, el infinitivo (V) ha sido desplazado junto con su complemento (X), lo cual indica que X y V - mayor procurar - son un constituyente. Este ejemplo, por lo tanto, sugiere que la promoción de clíticos medieval no necesariamente supone la restructuración de la construcción de infinitivo.

\subsection{El caso de la intercalación de constituyentes}

Como resaltan varios autores, la promoción de clíticos en el español actual así como en el portugués actual exige la adyacencia del verbo regente y del infinitivo, es decir, a excepción de ciertas preposiciones y partículas, no puede aparecer prácticamente ningún elemento entre los dos verbos de la construcción. ${ }^{5}$ Esta restricción aparentemente apoya la teoría de la restructuración puesto que la intercalación de constituyentes como el sujeto y de complementos del infinitivo implica que los dos verbos no constituyan una unidad sintáctica y por lo tanto bloquea la promoción de clíticos, como se ve en (6).

(6) a. esp. act. *Te quiere mi hermano llamar.

b. ptg. act. *Quer-te o meu irmão chamar.

c. esp. act. ?Quiere mi hermano llamarte.

d. ptg. act. ?Quer o meu irmão chamar-te.

e. esp. act. * Le quiero el libro dar.

f. ptg. act. *Quero-lhe o livro dar.

\footnotetext{
${ }^{4}$ Los argumentos de Rizzi (1982:38) se basan principalmente en la separabilidad de los componentes del complejo verbal: "If the verbal complex were dominated by the lexical category $\mathrm{V},[\ldots]$ we would expect that no other lexical material could intrude within its elements, this being a trivial property of lexcial categories. However, this prediction is false: many kinds of adverbs can break into the verbal complex". Su conclusión es que el complejo verbal restructurado no funciona como un verbo: "If this is correct, we are led to conclude that Italian syntax makes use of a syntactic category, distinct from $\mathrm{V}$, dominating nonlexical verbal compounds".

${ }^{5}$ Las observaciones de Bolinger (1949); Bok-Bennema \& Croughs-Hageman (1980); Fernández (2000:\$19.5.5.); Luna Traill (1972); Pizzini (1982) referentes al caso del español son unánimes desde este punto de vista. En relación con el portugués actual confirman la necesidad de adyacencia Pizzini (1981:417) y Salvi (1991b:164-166), aunque éste aduce contraejemplos también.
} 
g. $\quad$ esp. act. *Quiero el libro darle.

h. ptg. act. *Quero o livro dar-lhe.

Se puede objetar que, según se ve en $(6 \mathrm{~g}-\mathrm{h})$, la intercalación del argumento interno entre los dos verbos es agramatical también cuando no se produce la promoción de clíticos, o sea, la agramaticalidad de $(6 g-h)$ se debe a esta misma intercalación y no depende de la colocación de los clíticos. Sin embargo, (6cd) - con sujeto intercalado y clíticos no promovidos - son aceptables en el lenguaje coloquial, lo cual significa que la agramaticalidad de (6a-b) se debe a que la promoción de clíticos es incompatible con la intercalación del sujeto.

Rivero (1993:118) observa que, a diferencia de la situación actual, ilustrada en (6), en el español antiguo en los casos de promoción, “[...] el material que puede intervenir era mucho más variado que actualmente". El análisis de los textos medievales confirma dicha afirmación no sólo en el caso del español sino también en relación con el portugués. El corpus medieval en ambos idiomas contiene numerosos casos en los que el elemento adverbial intercalado entre los dos verbos no impide la promoción de clíticos; cf. (7a-b). La intercalación del sujeto ha sido registrada con promoción de clíticos en los textos medievales analizados procedentes de ambas lenguas - cf. (7c-f). En los textos portugueses, además, se puede observar también la posibilidad de intercalar complementos regidos por el infinitivo - cf. $(7 \mathrm{~g}-\mathrm{h})-\mathrm{e}$ incluso la intercalación simultánea del sujeto pronominal y del complemento regido cf. (7i).

(7) a. esp. ant.[.. .] bien me cueydo agora vengar de vos. (VEI, 38)

b. ptg. ant. [...] que se os no $\langle\mathrm{m}\rangle$ podessem e $\langle\mathrm{m}\rangle$ esse dya ve $\langle\mathrm{n}\rangle$ cer [...] (CGE, 53)

c. esp. ant. [...] deuel el merino coger sus derechos [...] (FUE, 37)

d. esp. ant. Et deuel el Rey justiciar el cuerpo por este fecho. (FUE, 83)

e. ptg. ant. [...] a que sse deue $\langle\mathrm{m}\rangle$ os home $\langle\mathrm{n}\rangle \mathrm{s} \operatorname{co}\langle\mathrm{n}\rangle \mathrm{ffessar}[\ldots]$ (SET, 140)

f. ptg. a. Ca $a$ nom quer niuu $\langle\mathrm{m}\rangle$ provar [...] (GRA, 32)

g. ptg. ant. nam se podia a el chegar niuu $\langle\mathrm{m}\rangle$ que se nam queimasse. (GRA, 25)

h. ptg. ant. E fezeo consigo levar muy honrradame〈n〉te (CGE, 44)

i. [...] tam bem o poderedes vós a mim dizer como eu a vós. (GRA, 42)

En relación con los ejemplos presentados en (7) conviene hacer algunas observaciones. Aunque la posibilidad de intercalar elementos entre las dos formas verbales es un factor que distingue la situación medieval de la actual, 
cabe señalar que la adyacencia de los dos verbos no es exigida por la teoría de la restructuración. Según explica Rivero (1993:124), "los adverbios o los complementantes pueden formar parte del complejo restructurado", así que los ejemplos presentados en $(7 \mathrm{a}-\mathrm{b})$, con elementos adverbiales intercalados, son compatibles con la teoría de Rizzi (vid. supra, nota 4). En conformidad con esta situación, sólo los ejemplos que aparecen en $(7 \mathrm{c}-\mathrm{i})$ pueden tenerse en cuenta como contraejemplos potenciales de la teoría de la restructuración. Además, es preciso llamar la atención también sobre el hecho de que, aunque todos los ejemplos aducidos en (7) coinciden en contener constituyentes intercalados entre el verbo regente y el infinitivo, en realidad dicha intercalación es manifestación de fenómenos sintácticos distintos. Tal como se propone en Berta (2001:39), la intercalación del sujeto, ilustrada en (7c-f), debe ser interpretada como una posibilidad asegurada por el sistema llamado V2, conocido en las lenguas romances medievales, que permitía que el sujeto siguiera al verbo si otro constituyente lo precedía. También era posible la posposición del sujeto cuando el verbo se colocaba al comienzo de la frase (orden V1). Así, cuando el sujeto medieval sigue a un verbo que rige un infinitivo, puede situarse entre las dos formas verbales separándolas. La intercalación de los complementos regidos por el infinitivo, sin embargo, se debe a otro fenómeno sintáctico, en concreto a la conservación del antiguo orden SOV, en el que los complementos podían preceder al verbo que los regía. De acuerdo con ello, en $(7 \mathrm{~g}-\mathrm{h})$ los complementos regidos por el infinitivo se colocan entre éste y el verbo supraordinado. Nótese que la colocación simultánea del sujeto y del complemento del infinitivo entre las dos formas verbales en nuestro (7i) se debe a la manifestación simultánea de ambos fenómenos mencionados: el sujeto - vós - se intercala como consecuencia del sistema V2, mientras que el objeto indirecto - a mim - se intercala porque dentro del grupo del infinitivo regido el orden de los constituyentes es OV.

La distinción de los tipos de intercalación también es importante desde el punto de vista de la aplicabilidad de la teoría de la restructuración porque los constituyentes de categoría diferente se comportan de manera distinta. Frente a los elementos adverbiales intercalados, los cuales, como se ha señalado arriba, son compatibles con la restructuración, los argumentos internos intercalados bloquean la formación de un complejo verbal restructurado. Esta diferencia se ilustra en las fórmulas que se proponen en (8a) - donde Adv es el elemento adverbial intercalado - y (8b) - donde X es el argumento interno intercalado. Tal interpretación significa que aunque los ejemplos presentados en $(7 \mathrm{a}-\mathrm{b})$ no descartan la condición de la formación de un complejo verbal restructurado, los de $(7 \mathrm{~g}-\mathrm{i})$ demuestran que la promoción de clíticos medieval es posible también en aquellos casos en los que la restructuración no se puede producir. A diferencia de estos dos casos bastante claros, la intercalación del sujeto, debida al funcionamiento del sistema V2 - vid. supra - es un 
caso problemático. Rivero no menciona que el sujeto pueda formar parte del complejo restructurado, por lo tanto se puede suponer - como se hace en Berta (2001:38-39) - que su intercalación impide la restructuración. De esta manera los ejemplos medievales de $(7 \mathrm{c}-\mathrm{f})$ demostrarían que la promoción de clíticos se puede realizar sin que se haya producido la formación de un complejo verbal. Sin embargo, la intercalación del sujeto puede tener una explicación compatible con la restructuración si se supone que el verbo regente puede desplazarse hacia la izquierda después de haberse realizado la formación del complejo verbal restructurado. Tal hipótesis coincide con la idea ya mencionada de que dicho complejo verbal no se comporta como un verbo único, por lo tanto sus componentes - tanto el verbo regente como el infinitivo regido (vid. supra, en 2.1.) - pueden extraerse de ella independientemente. En (8c) se propone una fórmula que ilustra primero la formación de complejo verbal - [Aux V] - y después el desplazamiento del verbo regente - representado por Aux - hacia la izquierda así dejando el sujeto - representado por S - entre las dos formas verbales.
(8) a. Aux $[\mathrm{Adv} V] \rightarrow[\operatorname{Aux} \mathrm{Adv} V]$
b. Aux $[\mathrm{X} \mathrm{V}] \rightarrow *[\operatorname{Aux} \mathrm{X} V]$
c. $\mathrm{S}$ Aux $[\mathrm{V}$ X] $\rightarrow \mathrm{S}$ [Aux V]X $\rightarrow$ Aux S [_V]X

En resumen, se puede concluir que, aunque la adyacencia léxica de las dos formas verbales de la construcción no es un factor decisivo desde el punto de vista de la teoría de la restructuración - con la cual la intercalación del sujeto y de elementos adverbiales es perfectamente compatible -, la existencia de ejemplos medievales que contienen argumentos internos intercalados entre el verbo regente y el infinitivo demuestra que en el período medieval la promoción de clíticos no exigía necesariamente la formación de un complejo verbal restructurado.

\subsection{El caso de dos verbos regentes}

En algunos casos el mismo infinitivo depende de dos - o más - verbos regentes distintos. Los ejemplos de (9), tomados de Rizzi (1982:11) y aplicados al español, demuestran que en estos casos en el español actual no se produce la promoción de clíticos.

(9) a. Mario debería - pero a mi parecer no podrá nunca - pagarle enteramente su deuda.

b. *Mario le debería - pero a mi parecer no le podrá nunca - pagar enteramente su deuda. 
Se supone que en casos como (9) ambos verbos regentes exigen la aparición de un infinitivo pero como los dos infinitivos regidos son idénticos, se elimina uno. Siguiendo a Rizzi se debe suponer que para que se pueda eliminar un elemento idéntico evitando su repetición, en este caso el infinitivo regido por ambos verbos regentes, este elemento debe ser un constituyente independiente como en (10a) y no parte de un constituyente como en (10b).

$$
\begin{aligned}
& \text { (10) a. } \quad \mathrm{V}[\mathrm{INFX}]+\mathrm{V}[\mathrm{INFX}] \rightarrow \mathrm{V}+\mathrm{V}[\mathrm{INFX}] \\
& \text { b. }[\mathrm{VINF}] \mathrm{X}+[\mathrm{VINF}] \mathrm{X} \rightarrow * \mathrm{~V}+[\mathrm{VINF}] \mathrm{X}
\end{aligned}
$$

Sin embargo, la promoción de clíticos en las variantes actuales de las lenguas analizadas exige la estructura (10b), en la que el verbo regente y el infinitivo forman una unidad verbal compleja, es decir, un único constituyente, por eso la eliminación del infinitivo repetido es incompatible con la promoción de clíticos, es decir, con la restructuración. En los textos antiguos, en cambio, se documentan algunos casos en los que el infinitivo complemento depende de dos verbos regentes sin que esta situación bloquee la promoción de clíticos, como se ve en (11).

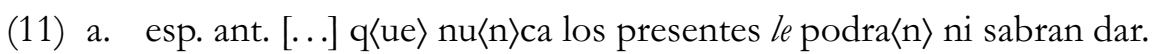
(ARA, 5)

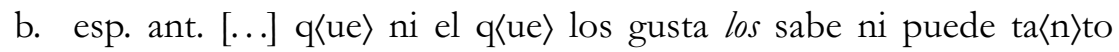
encareçer como deue (ARA, 31)

c. esp. ant. [...] ni el quue $\rangle$ dellos carece los puede ni sabe creer como $\mathrm{cu}\langle\mathrm{m}\rangle$ ple. (ARA, 31)

d. esp. ant. qua $\langle\mathrm{n}\rangle \mathrm{do}$ y por do $\langle\mathrm{n}\rangle \mathrm{de} l o$ pueda $\langle\mathrm{n}\rangle$ y deua $\langle\mathrm{n}\rangle$ mejor fazer [...] (ARA, 14)

e. ptg. ant. [...] maas que ante as no $\langle\mathrm{m}\rangle$ saberia ne $\langle\mathrm{m}\rangle$ poderia penssar. (SET, 135)

Siguiendo la argumentación anterior, estos hechos también apoyan la idea de que, según el testimonio de las fuentes medievales, en las variantes antiguas del español y del portugués la promoción de clíticos no exige la restructuración de las construcciones de infinitivo. Se debe reconocer, por otra parte, que interpretando los dos verbos regentes como una unidad sintáctica que rige un infinitivo, - suponiendo una estructura como $[\mathrm{V}+\mathrm{V}] \mathrm{INF}-$, no sería necesario suponer la eliminación del infinitivo. Si se tiene en cuenta que los dos verbos que rigen el mismo infinitivo son casi siempre los mismos, - poder y saber, usados antiguamente casi como sinónimos según Yllera (1980:137) - se puede confirmar tal interpretación y entonces los ejemplos aducidos en (11) no deben tenerse en cuenta como pruebas en contra de la teoría de la restructuración. 


\section{CONCLUSIONES}

En las líneas precedentes se ha examinado la estructura de las construcciones de infinitivo en las variantes medievales y actuales del español y del portugués, teniendo en cuenta principalmente la posibilidad de aplicar la teoría de la restructuración como posible explicación del fenómeno de la promoción de clíticos. La comparación de los ejemplos modernos con frases procedentes de un corpus medieval ha demostrado que mientras que en las variantes actuales de dichas lenguas iberorrománicas la promoción de clíticos exige que el verbo regente y el infinitivo formen una unidad verbal compleja, según el testimonio de los textos medievales analizados en el período medieval tal restructuración de la construcción no era condición de la promoción de clíticos. Esta conclusión se basa en el análisis de ejemplos con infinitivos complementos antepuestos al verbo regente, constituyentes regidos intercalados e infinitivos complementos regidos por varios verbos supraordinados, el cual demuestra que en los textos medievales la promoción de clíticos se documenta también en construcciones en las cuales se debe excluir la restructuración.

Aunque los resultados del análisis realizado apoyan nuestra hipótesis inicial - consistente en que las construcciones medievales y actuales deben de tener estructura distinta -, las conclusiones arriba formuladas hacen surgir una contradicción que todavía se debe solucionar. Como se ha mencionado en la Introducción, el punto de partida para el análisis de Rizzi era que los clíticos deben encontrarse dentro de la misma frase en la que se sitúa el infinitivo. También Salvi (1991a;b), al tratar de las reglas de la colocación de los clíticos medievales en portugués afirma que el clítico medieval se coloca inmediatamente detrás del primer elemento (o constituyente) de la frase en la que se encuentra. Estas hipótesis implican que el clítico no puede abandonar la frase del infinitivo, por lo cual en los casos de promoción el verbo regente y el infinitivo deben pertenecer a la misma frase, o sea, la promoción de clíticos exige que la construcción sea "monofrástica". El problema consiste en que, frente a las variantes actuales de los idiomas examinados, en las que la restructuración es aplicable como explicación de la formación de la estructura "monofrástica", según nuestro análisis en sus variantes medievales se pueden aducir varios ejemplos en los que, aunque la promoción de clíticos indica que la construcción tiene que ser "monofrástica", ciertas circunstancias sintácticas demuestran que no se puede aplicar la restructuración. Esta situación aparentemente contradictoria significa que para explicar la colocación de los clíticos en los casos de promoción, se debe buscar una solución diferente de la teoría de la restructuración.

Un posible análisis que puede resolver el problema arriba mencionado puede ser el que se propone en Berta (2001:42-43), que, aplicando la teoría de Pearce, elaborada para el caso del francés antiguo, atribuye al infinitivo de- 
pendiente un rango inferior al de frase, suponiendo que la unidad formada por el infinitivo y sus complementos no tiene la autonomía de una frase, sino que se comporta como un sintagma - que se puede llamar grupo verbal $(\mathrm{GV})$ - regido por el verbo estructuralmente supraordinado. La ventaja de este análisis es que se puede aplicar tanto en casos semejantes a (12a), en que la restructuración se debe excluir como en casos similares a $(12 \mathrm{~b}-\mathrm{c})$, en los que la restructuración también sería aplicable.

(12) a. [F fezeo [Gv [ consigo levar] [ muy honrradame〈n〉te ] ] ]

b. [F quiero gelo [ GV dar ] ]

c. [F quero-lhe [Gv tolher a coroa ] ]

Considerando que el constituyente cuyo núcleo es el infinitivo regido no tiene el rango de frase, la estructura de las construcciones con promoción de clíticos, de acuerdo con Benucci (1990) será "monofrástica" pero sin tener que suponer la restructuración propuesta por Rizzi para la situación actual.

TEXTOS ANALIZADOS

Textos españoles

ADMYTE (1995) = Archivo Digital de Manuscritos y Textos Españoles. Madrid: Micronet S.A.

ARA (1995) = Gauberto Fabricio de Vagad, Crónica de Aragón. ADMYTE, CD-ROM, disco 1.

FUE (1995) = Fueros de Castilla. ADMYTE, CD-ROM, disco 0.

ULT $(1995)=$ Gran conquista de Ultramar. ADMYTE, CD-ROM, disco 0.

VEI $(1995)=$ Crónica de veinte reyes. ADMYTE, CD-ROM, disco 0.

Textos portugueses

CGE (1959) = Crónica Geral de Espanha de 1344. Edição crítica do texto português. Ed. de L. F. Lindley Cintra, vol. III, Lisboa, Imprensa Nacional/Casa da Moeda.

SET $(1980)=$ Alphonse X: Primeyra Partida. Édition et Étude. Ed. de J. de Azevedo Ferreira, Braga, Instituto Nacional de Investigação Científica.

GRA $(1995)=$ A Demanda do Santo Graal. Ed. de I. Freire Nunes, Lisboa, Imprensa Nacional/Casa da Moeda.

BIBLIOGRAFÍA

Benucci, F. (1990): Destrutturazione. Classi verbali e costruzioni perifrastiche nelle lingue romanze antiche e moderne. Unipress, Padova.

Berta, T. (2000a): La posición de los pronombres clíticos españoles en construcciones con infinitivo. In: Franco Figueroa, M., et al. (eds.) Nuevas perspectivas en la enseñanza del español como lengua extranjera. Actas del X Congreso Internacional de la Asociación para la Enseñanza del Español como Lengua Extranjera (ASELE), 22-25 de septiembre de 1999. Vol. 1, Servicio de Publicaciones de la Universidad de Cádiz, Cádiz. pp. 123-132. 
Berta, T. (2000b): La subida de clíticos en español medieval y en español moderno. Acta Univ. Szegediensis, Acta Hispanica, 5: 83-99.

Berta, T. (2001): La estructura de las construcciones de infinitivo en textos españoles y portugueses medievales. In: Kiss, S., Salvi, G., Sziji, I. (eds.) rom.hu. Études de linguistique romane, Ibisz/Programme doctoral de linguistique romane de l'Université Eötvös Loránd, Budapest. pp. 33-47.

Bok-Bennema, R., Croughs-Hageman, A. (1980): La subida de los clíticos en castellano. In: Diálogos hispánicos de Amsterdam I. Los clíticos en el español actual, Universiteit von Amsterdam, Amsterdam. pp. 63-92.

Bolinger, D. L. (1949): Discontinuity of the Spanish conjunctive pronoun. Language, 25 : 253260.

Colburn, G. B. (1928): The complementary infinitive and its pronoun object. Hispania, 11 : 424-429.

Davies, M. (1995): Analyzing syntactic variation with computer-based corpora: the case of modern Spanish clitic climbing. Hispania, 78:370-380.

Davies, M. (1997a): A corpus-based approach to diachronic clitic climbing in Portuguese. Hispanic Journal, 17:93-111.

Davies, M. (1997b): The evolution of Spanish clitic climbing: a corpus-based approach. StN, $69: 251-263$.

Fernández, O. (ed.) (1993): Los pronombres átonos. Santillana, Taurus, Madrid.

Fernández, O. (2000): El pronombre personal. Formas y distribuciones. Pronombres átonos y tónicos. In: Bosque, I., Demonte, V. (eds.) Gramática descriptiva de la lengua española, Espasa, Real Academia Española, Madrid. pp. 1262-1269.

Luján, M. (1993): La subida de clíticos y el modo verbal en los complementos verbales del español. In: Fernández (1993:235-282).

Luna Traill, E. (1972): Sobre la sintaxis de los pronombres átonos en construcciones de infinitivo. Anuario de Letras, $10: 191-200$.

Pizzini, Q. A. (1981): The placement of clitic pronouns in Portuguese. Linguistic Analysis, 8 : 403-430.

Pizzini, Q. A. (1982): The positioning of clitic pronouns in Spanish. Lingua, 57:47-69.

Quicoli, A. C. (1976): Conditions on Clitic-Movement in Portuguese. Linguistic Analysis, 2: 199-221.

Rivero, M.-L. (1991): Clitics and NP climbing in Old Spanish. In: Gil, F. M., Campos, H. (eds.) Current Studies in Spanish Linguistics, Georgetown Univ. Press, Washington D. C. pp. 241-282.

Rivero, M.-L. (1993): Subida de Clíticos y de SN en español antiguo. In: (Fernández 1993: 101-136).

Rizzi, L. (1982): Issues in Italian syntax. Foris, Dordrecht.

Salvi, G. (1991a): Difesa e illustrazione della Legge di Wackernagel applicata alle lingue romanze antiche: la posizione delle forme pronominali clitiche. In: Miscellanea G. B. Pellegrini, Unipress, Padova. pp. 439-462.

Salvi, G. (1991b): La struttura della frase e l'ordine delle parole in galego-portoghese. Tesis de candidatura a doctor académico, Universidad Loránd Eötvös, Budapest.

Spaulding, R. K. (1927): Puedo hacerlo versus lo puedo hacer and similar cases. Hispania, 10 : 343-348.

Wanner, D. (1982): A history of Spanish clitic movement. In: Macaulay, M., et al. (eds.) Proceedings of the 8th Annual Meetning of the Berkeley Linguistic Society, Berkeley Linguistic Society, Berkeley. pp. 135-147.

Yllera, A. (1980): Sintaxis histórica del verbo español: las perífrasis medievales. Universidad de Zaragoza, Zaragoza. 